\title{
Minimization of Impulse Response of Dynamic Structures Using A Ground-Hooked Dynamic Vibration Absorber
}

\section{You Lin Cheung and Waion Wong*}

Department of Mechanical Engineering, The Hong Kong Polytechnic University, Hong Kong SAR, China

\begin{abstract}
A non-traditional dynamic vibration absorber (DVA) with ground-support is proposed to minimize the impulse response of a single degree-of-freedom (SDOF) system. The conventional wisdom of suppressing random vibration of a machine is to add a damper to its mounting or to increase the mass of the primary system. We have proved that the proposed DVA with ground-support has better suppression of the impulse response of a single degree-of-freedom system than just using the damper alone or by increasing the mass of the primary system. Design guidelines to the engineer to apply the proposed DVA with ground-support for suppressing the impulse response of a single degree-of-freedom system are provided.
\end{abstract}

\section{Introduction}

Dynamic vibration absorber (DVA) is an auxiliary sprung mass system which, when correctly tuned and attached to a vibrating system subject to harmonic excitation, causes to cease the steadystate motion at the point to which it is attached. The DVA needed to be tuned properly such that it can reduce the vibration amplitude of the controlled system. The first research conducted at the beginning of the twentieth century considered an undamped DVA tuned to the frequency of the disturbing force by Frahm [1]. Such an absorber is a narrow-band type as it is unable to eliminate structural vibration of the controlled system after any change of the disturbing frequency. A damper is often added to the DVA in order to widen the bandwidth of its operation. Ormondroyd and Den Hartog [2,3] proposed the optimization principle of the damped DVA in terms of minimizing the resonant amplitude response of the primary system, which called $H \infty$ optimization of DVA. Crandall and Mark [4] proposed another optimization principle of the damped DVA in terms of minimizing the total vibration energy or the mean square motions of the primary structure under white noise excitation, which called $\mathrm{H}_{2}$ optimization of dynamic vibration absorber. Since an impulse input in time domain is equivalent to white noise excitation in frequency domain, $\mathrm{H} 2$ optimization of the DVA would be more useful to suppress impulse response or random vibration of the primary system. The exact solution of the $\mathrm{H}_{2}$ optimization for the traditional DVA attached to an undamped primary system was derived by Warburton [5-7].

A non-traditional damped DVA with ground support as shown in Fig.1was proposed by Ren [8], and Liu [9] recently. Based on the fixed-points theory [3], the $H \infty$ optimum tuning parameters of such a vibration absorber has been derived analytically for suppressing the resonant vibration of a SDOF system subjected to force excitation [8-9] or caused by ground motions[10]. In this article, this non-traditional damped DVA is proposed to minimize the impulse response of a single degree-of-freedom system. The conventional wisdom of suppressing random vibration of a machine is to add a damper to its mounting or to increase the mass of the dynamic system. As shown in the following section, it is proved that the proposed DVA with ground-support has better suppression of the impulse response of a single degree-of-freedom system than just using the damper alone or by increasing the mass of the controlled system.

\section{Theory}

A non-traditional design of DVA with ground support [8,9] as shown in Figure 1 is called Model A in the following discussion.
It is compared to a ground-hooked damper and an added mass for the suppression of impulsive force response of a SDOF system in the following. The frequency response function of the mass $\mathrm{M}$ of Model $\mathrm{A}$ can be derived and written as [8].

$$
|=|=\mid=\frac{\mu\left(\gamma^{2}-\lambda^{2}\right)+\zeta \lambda}{\mu\left[\left(1-\lambda^{2}\right)\left(\gamma^{2}-\lambda^{2}\right)\right]+2 \zeta\left(1-\lambda^{2} \mu \gamma^{2}\right)}
$$

where $\mu=\frac{m}{M}, \omega_{a}=\sqrt{\frac{k}{m}}, \omega_{n}=\sqrt{\frac{K}{M}}, \gamma=\frac{\omega_{a}}{\omega_{n}}$ and $j=\sqrt{-1 .}$

Consider Model B as shown in Figure 2 which is a damped SDOF system with the same amount of damping of Model A. The frequency response of mass M of Model B may be written as

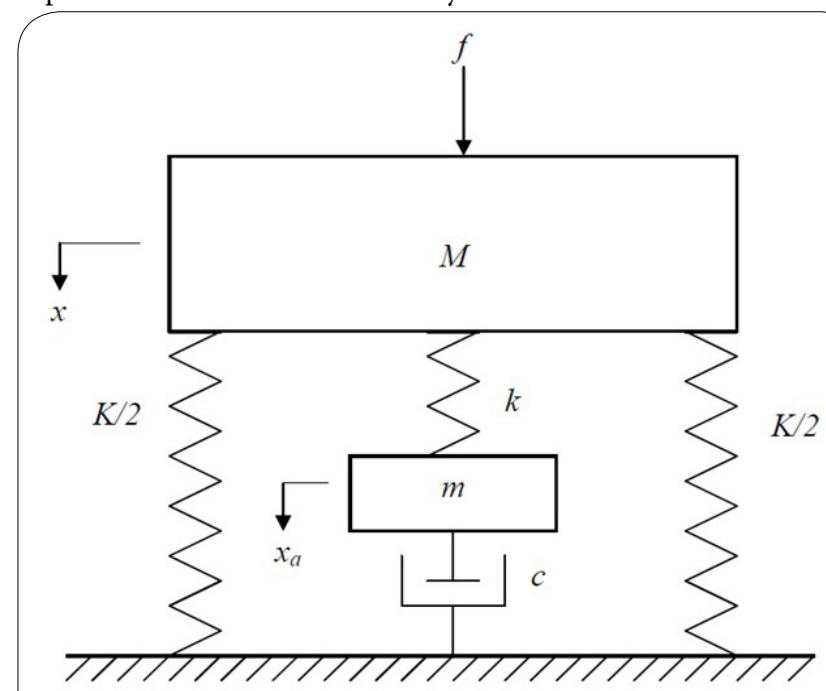

Figure 1: Schematic diagram of model A: the non-traditional dynamic vibration absorber ( $\mathrm{m}$-k-csystem) attached to the primary (M-K) system.

"Corresponding Author: Dr. Waion Wong, Department of Mechanical Engineering, The Hong Kong Polytechnic University, Hong Kong SAR, China, ; E-mail: mmwowong@polyu.edu.hk

Citation: Cheung YL, Wong W (2016) Minimization of Impulse Response of Dynamic Structures Using A Ground-Hooked Dynamic Vibration Absorber. Int J Mech Syst Eng 2: 113. http://dx.doi.org/10.15344/2455-7412/2016/113

Copyright: ( 2016 Sassi et al. This is an open-access article distributed under the terms of the Creative Commons Attribution License, which permits unrestricted use, distribution, and reproduction in any medium, provided the original author and source are credited. 
Citation: Cheung YL, Wong W (2016) Minimization of Impulse Response of Dynamic Structures Using A Ground-Hooked Dynamic Vibration Absorber. Int J Mech Syst Eng 2: 113. http://dx.doi.org/10.15344/2455-7412/2016/113

Page 2 of 3

Consider Model B as shown in Figure 2 which is a damped SDOF system with the same amount of damping of Model A. The frequency response of mass $\mathrm{M}$ of Model B may be written as $\left|\frac{X_{1}}{F / K}\right|_{B}=\left|G_{B}\right|=\frac{1}{1-\lambda^{2}+2 j \zeta \lambda}$.

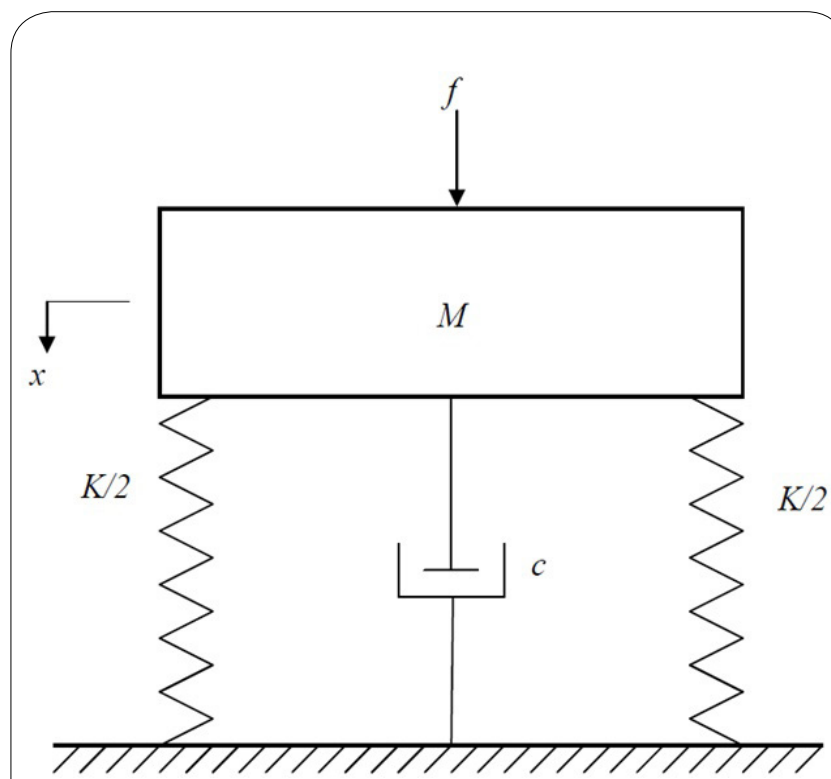

Figure 2: Schematic diagram of model B: a SDOF vibrating system with primary damping of damping coefficient $\mathrm{c}(\mathrm{M}-\mathrm{K}-\mathrm{c})$ system.

Consider Model $\mathrm{C}$ as shown in Figure 3 which is a damped SDOF system with the same amount of damping of Model A and an added mass $\mathrm{m}$. The frequency response of Model $\mathrm{C}$ may be written as

$$
\left|\frac{X}{F / K}\right|_{c}=\left|G_{c}\right|=\frac{1}{1-(1+\mu) \lambda^{2}+2 j \zeta \lambda}
$$

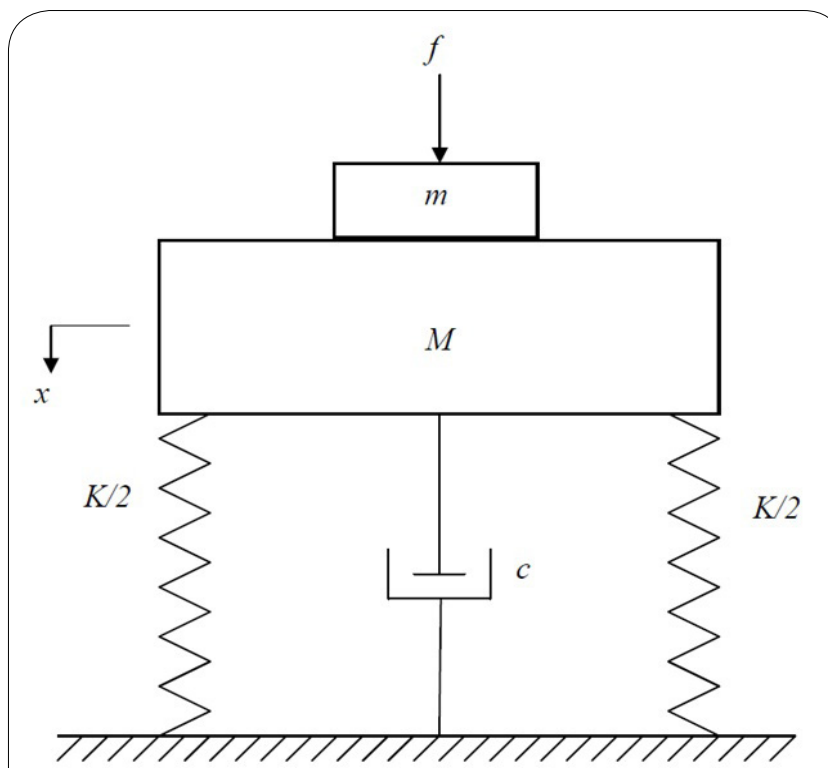

Figure 3: Schematic diagram of model C: a SDOF vibrating system with primary damping of damping coefficient cand anadded massm $(m-M-$ $K-c)$ system.
The mean square motion of the primary mass $\mathrm{M}$ of the three models may be written as [11]

$$
E\left[x^{2}\right]=E\left[|G|^{2}\right]=\int_{-\infty}^{\infty}|G|^{2} S_{y}(\omega) d \omega
$$

Where $\mathrm{G}$ is the frequency response function of the primary mass and $S_{y}(\omega)$ is the input mean square spectral density function.

If the input spectrum is assumed to be ideally white, i.e. $S_{y}(\omega)=S_{0}$, a constant for all frequencies, the integral of Eq.(4) can then be reduced to

$$
E\left[x^{2}\right]=S_{0} \int_{-\infty}^{\infty}|G(\omega)|^{2} d \omega
$$

Using Eq.(5), the non-dimensional mean square motion can be defined as

$$
E\left[x^{2}\right]=\omega_{n} S_{0} \int_{-\infty}^{\infty}|G(\lambda)|^{2} d \lambda .
$$

A useful formula of Crandall [12] written as Eq. (7)below is used for solving Eq.(6).

$$
\text { if } G(\omega)=\frac{-j \omega^{3} B_{3}-\omega^{2} B_{2}+J \omega B_{1}+B_{0}}{\omega^{4} A_{4}-j \omega^{3} A_{3-} \omega^{2} A_{2}+j \omega A_{1}+A_{0}}
$$

Then

$\int_{-\infty}^{\infty}|G(\omega)|^{2} d \omega$

$$
=\frac{\pi\left[\frac{B_{0}^{2}\left(A_{2} A_{3}-A_{1} A_{4}\right)}{A_{0}}+A_{3}\left(B_{1}^{2}-2 B_{0} B_{2}\right)+A_{1}\left(B_{2}^{2}-2 B_{1} B_{3}\right)+\frac{B_{3}^{2}\left(A_{1} A_{2}-A_{0} A_{3}\right)}{A_{4}}\right]}{A_{1}\left(A_{1} A_{2}-A_{0} A_{3}\right)-A_{0} A_{3}^{2}}
$$

Comparing Eqs.(1) and (7), we may write

$A_{0}=\mu \gamma^{2}, A_{1}=2 \zeta\left(1+\mu \gamma^{2}\right), A_{2}=\mu\left(1+\gamma^{2}+\mu \gamma^{2}\right), A_{3}=2 \zeta, \quad A_{4}=\mu$,

$B_{0} \mu \gamma^{2}, B_{1}=2 \zeta, \quad B_{2}=\mu, B_{3}=0$.

Using Eqs.(6) and (8), the mean square motion of the primary structure with the proposed DVA may be written as

$$
\begin{aligned}
& E\left[\left|G_{a}\right|^{2}\right]=\frac{\pi \omega_{n} S_{0}}{2 \zeta}\left(1+\frac{\mu-2}{\gamma^{2}}+\frac{4 \zeta^{2}+\mu^{2}}{\mu^{2} \gamma^{4}}\right) \\
& \frac{\partial E\left[\left|G_{a}\right|^{2}\right]}{\partial \gamma}=\frac{\pi \omega_{n} S_{0}}{2 \zeta}\left(\frac{-2(\mu-2)}{\gamma^{3}}-\frac{4 \zeta^{2}+\mu^{2}}{\mu^{2} \gamma^{5}}\right)=0 .
\end{aligned}
$$

Using Eq. (10), the optimum tuning ratio may be written as

$$
\gamma_{\text {opt }}=\sqrt{\frac{8 \zeta^{2}+2 \mu^{2}}{\mu^{2}(2-\mu)}}, \mu<2
$$

Substituting Eq. (11) into Eq. (6), the mean square motion of the mass $M$ of Model A may be written as

$$
E\left[\left|G_{A}\right|^{2}\right]=\frac{\pi \omega_{n} S_{0}}{2 \zeta}\left[1-\frac{\mu^{2}(2-\mu)^{2}}{4\left(4 \zeta^{2}+\mu^{2}\right)}\right] .
$$

A useful formula of Crandall [12] written as Eq. (13) below is used for solving Eq. (7).

$$
G(\omega)=\frac{i \omega B_{1}+B_{0}}{-\omega^{2} A_{2}+i \omega A_{1}+A_{0}} \text { then } \int_{-\infty}^{\infty}|G(\omega)|^{2} d \omega=\pi\left[\frac{\frac{A_{2} B_{0}^{2}}{A_{0}}+B_{1}^{2}}{A_{1} A_{2}}\right] \text {. }
$$


Citation: Cheung YL, Wong W (2016) Minimization of Impulse Response of Dynamic Structures Using A Ground-Hooked Dynamic Vibration Absorber. Int J Mech Syst Eng 2: 113. http://dx.doi.org/10.15344/2455-7412/2016/113

Page 3 of 3

Comparing Eqs. (2) and (13)for Model B, we have

$$
A_{0}=1, \quad A_{1}=2 \zeta, \quad A_{2}=1, B_{0}=1, \quad B_{1}=0 .
$$

Substituting Eq. (14) into Eq. (13), the mean square motion of the mass $\mathrm{M}$ of Model B may be written as

$$
E\left[\left|G_{b}\right|^{2}\right]=\frac{\pi \omega_{n} S_{0}}{2 \zeta}
$$

Comparing Eqs. (3) and (13)for Model C, we have

$$
A_{0}=1, \quad A_{1}=2 \zeta, \quad A_{2}=1+\mu, B_{0}=1, \quad B_{1}=0 .
$$

Substituting Eq. (16) into Eq. (13), the mean square motion of the mass $\mathrm{M}$ of Model $\mathrm{C}$ may be written as

$$
E\left[\left|G_{C}\right|^{2}\right]=\frac{\pi \omega_{n} S_{0}}{2 \zeta}
$$

As shown in Eqs. (15) and (17), the primary mass Min Model B and Model $\mathrm{C}$ have the same mean square motion response to an impulsive force input. It shows that increasing the mass of a damped SDOF system cannot reduce its mean square motion response to impulsive force excitation. Using Eqs (12) and (15), the ratio between the mean square motion of the primary structure with the proposed DVA and with a damper may be written as

$$
\frac{E\left[\left|G_{A}\right|^{2}\right]}{E\left[\left|G_{B}\right|^{2}\right]}=1-\frac{\mu^{2}\left(2-\mu^{2}\right)}{4\left(4 \zeta^{2}+\mu^{2}\right)} \leq 1
$$

Eq. (18) shows that $E\left[\left|G_{A}\right|^{2}\right]<E\left[\left|G_{B}\right|^{2}\right] I F \mu>0$. Therefore the mean square motion of the primary structure using the proposed DVA is smaller than that using only the damper. It shows that for any damped SDOF system as illustrated in Figure 2, the impulse response of the primary mass can be further reduced by using the proposed DVA with ground support if the DVA is tuned with $Y$ according to Eq. (11). $\frac{E\left[\left|G_{A}\right|^{2}\right]}{E\left[\left|G_{B}\right|^{2}\right]}$ is calculated according to Eq. (18) with different values of mass ratio and damping ratio and the results are plotted in Fig. 4 to show the performance of the proposed DVA in comparison of the primary damper.

A design guideline of applying the proposed DVA is suggested as follows. If an engineer plans to add a damper to reduce the dynamic response of a SDOF system subjected to impulsive force excitations, he or she may consider to use a sprung mass in series with the damper as illustrated in Fig. 1if possible. As shown in Eq. (18), the mean square motion response of the controlled system can be further reduced if the proposed DVA is used instead of the damper alone. The percentage of further reduction of mean square motion of the primary structure using the proposed DVA instead of just the damper alone can be estimated easily using Figure 4 after an applicable mass ratio is selected. The tuning frequency of the DVA can then be determined according to Eq. (11).

\section{Conclusion}

A dynamic vibration absorber with ground-support(Model A)is proposed to minimize the impulse response of a single degree-offreedom system. The conventional wisdom of suppressing random vibration of a machine is to add a damper to its mounting(Model B) or to increase the mass of the dynamic system (Model C). We have proved that the proposed DVA with ground-support has better suppression of the impulse response of a single degree-of-freedom

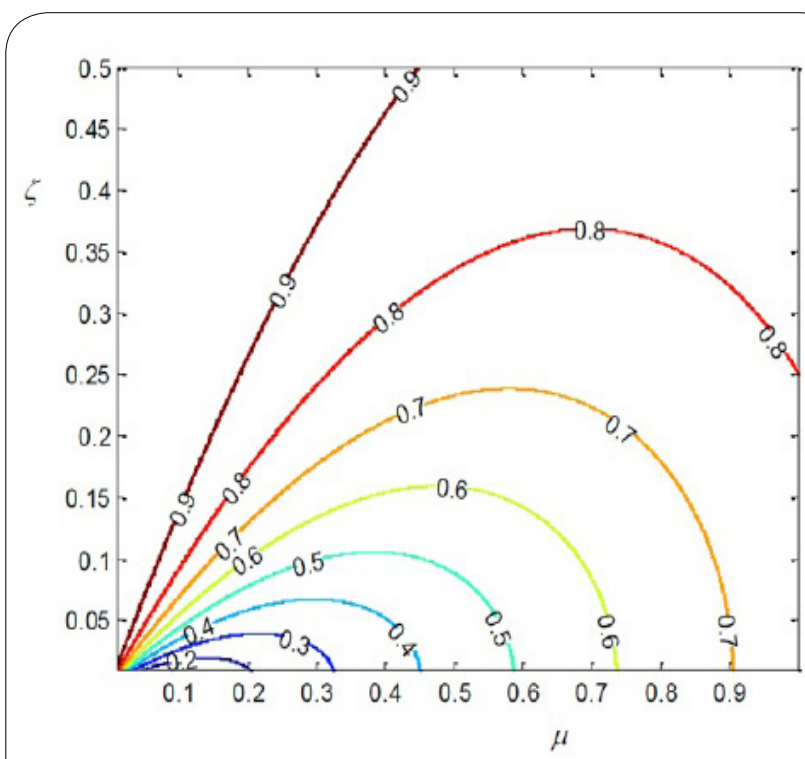

Figure 4: Comparison of the mean square motions of the mass

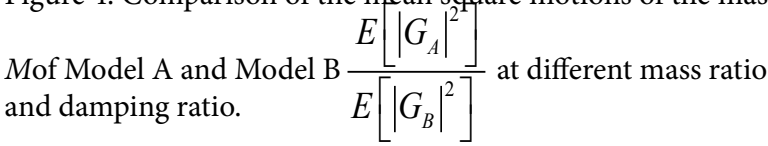

system than just using the damper alone or by increasing the mass of the dynamic system. As shown in the previous section, increasing the mass of a damped SDOF system cannot reduce its impulse response further. For example, if a machine is excited by impulsive force excitations and the engineer plans to add a damper to reduce its impulse response. We provided an alternative solution and design guidelines to the engineer to apply the proposed DVA for suppressing the impulse response of the machine.

\section{Competing Interests}

The authors have no competing interests with the work presented in this manuscript.

\section{References}

1. Frahm H (1911) Device for Damping Vibrations of Bodies, U.S. Patent, No. 989958: 3576-3580.

2. Ormondroyd J, Hartog JPD (1928) The theory of the dynamic vibration absorber, ASME J App Mech 50: 9-22.

3. Hartog JPD (1985) Mechanical Vibrations, Dover Publications Inc.

4. Crandall SH, Mark WD (1963) Random Vibration in Mechanical Systems, Academic Press.

5. Warburton GV, Ayorinde EO (1980) Minimizing structural vibrations with absorbers. Earthquake Engineering \& Structural Dynamics 8: 197-217.

6. Warburton GV (1981) Optimum absorber parameters for minimizing vibration response.Earthquake Engineering \& Structural Dynamics 9251 262.

7. Warburton GV (1982) Optimum absorber parameters for various combinations of response and excitation parameters, Earthquake Engineering \& Structural Dynamics 10: 381-401.

8. Ren MZ (2001) A variant design of the dynamic vibration absorber. J Sound Vibra 245: 762-770.

9. Liu K, Liu J (2005) The damped dynamic vibration absorbers: revisited and new result. J Sound Vibrat 284: 1181-1189. 
Citation: Cheung YL, Wong W (2016) Minimization of Impulse Response of Dynamic Structures Using A Ground-Hooked Dynamic Vibration Absorber. Int J Mech Syst Eng 2: 113. http://dx.doi.org/10.15344/2455-7412/2016/113

Page 3 of 3

10. Wong WO, Cheung YL (2008) Optimal design of a damped dynamic vibration absorber for vibration control of structure excited by ground motion. Engineer Struct 30: 282-286.

11. Asami T, Nishihara O, Baz AM (2002) Analytical Solutions to $\mathrm{H}_{\infty}$ and $\mathrm{H} 2$ Optimization of Dynamic Vibration Absorbers Attached to Damped Linear Systems, J Vibrat Acoustics 124: 284-295.

12. Gradshteyn IS, Ryzhik IM ( 1994) Table of Integrals, Series, and Products. Academic Press, Inc. 